\title{
Methanol poisoning induced acute onset parkinsonism, optic neuritis and peripheral neuropathy in a patient
}

Swayamsidha Mangaraj, Ganeswar Sethy, Ranjan Kumar Sen, Rabi Narayan Rout

Department of Internal Medicine, M. K. C. G. Medical College, Brahmapur, Odisha, India

Address for the Correspondence:

Dr. Swayamsidha Mangaraj, Department of Internal Medicine, Room No. 61, PG Hostel-2,

M. K. C. G. Medical College, Brahmapur - 760 004, Odisha, India.

E-mail: drsmangaraj@gmail.com

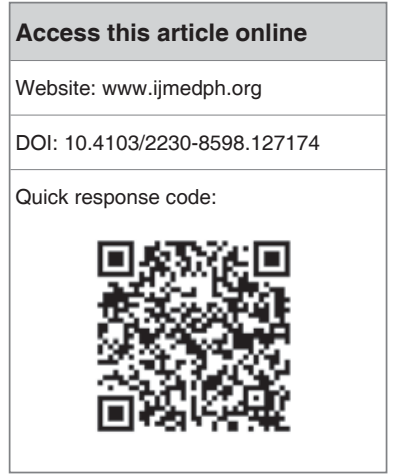

Methanol poisoning is a rare, but potentially life-threatening condition. It can result in significant neurological toxicities such as optic neuritis, encephalopathy, ataxia, polyneuropathy and parkinsonism. Concomitant presence of optic neuritis, parkinsonism and peripheral neuropathy in a patient of methanol intoxication has been very rarely reported in the literature. We report a case of a young male who developed acute onset parkinsonism, optic neuritis and peripheral neuropathy with evidence of putaminal necrosis following methanol poisoning. Early therapy can salvage a patient as well as minimize potentially catastrophic complications. Despite adequate management, a significant proportion of patients do develop devastating neurological deficits, which plague them for the rest of their lives.

Key words: Methanol poisoning, optic neuritis, parkinsonism, peripheral neuropathy, putaminal necrosis

\section{INTRODUCTION}

Methanol poisoning is a serious health hazard. This can prove fatal if appropriate therapy is not instituted at the earliest. Many serious neurological deficits can develop as a result of methanol intoxication. Optic atrophy leading to a variable degree of visual loss is commonly seen among survivors. ${ }^{[1]}$ Complications such as encephalopathy, polyneuropathy, ataxia and peripheral sensory neuropathy have been attributed to methanol intoxication. ${ }^{[1]}$ Rare cases of rapid onset parkinsonism following methanol poisoning have also been reported. ${ }^{[2]}$ There is a recent reported case where the patient developed optic neuritis, putaminal necrosis and polyneuropathy following methanol poisoning. ${ }^{[3]}$ Putaminal necrosis is a characteristic finding in cases of methanol intoxication. ${ }^{[4]}$ Clustering of all above deficits in a sole patient has very rarely been described.

\section{CASE REPORT}

A 26-year-old male, a manual laborer, presented to our tertiary care teaching hospital with the complaints of eight episodes of vomiting and blurring of vision for last 1 day. Patient had a history of binge alcohol intake 1 day back. The above symptoms started 6-8 h after the alcohol intake. There was no history of diarrhea, jaundice, fever associated with the symptoms. There was no history of hypertension, diabetes mellitus or any chronic illness.

On examination, the patient was dehydrated, drowsy and afebrile. He was tachypneic with a respiratory rate of $36 / \mathrm{min}$. He had a pulse rate of $94 / \mathrm{min}$ and a blood pressure $110 / 70 \mathrm{mmHg}$. Oxygen saturation was $96 \%$ on room air. There was no pallor cyanosis, clubbing, icterus or lymphadenopathy. Bilateral eyes were congested. Respiratory and cardiovascular examinations were normal. Neurological evaluation showed evidence of nystagmus bilaterally. Both pupils were dilated and showed sluggish light reflexes. Fundoscopy examination showed bilateral disc hyperemia and edema with the blurring of disc margins. Hematological evaluation including complete blood count revealed no abnormality. Random blood sugar, renal and hepatic function tests showed no abnormality. Biochemical evaluation showed a serum sodium-142 mEq/1, potassium-4 mEq/1, chloride-106 mEq/1. Arterial blood gas analysis revealed severe metabolic acidosis with pH-6.9 and bicarbonate level being $5 \mathrm{mEq} / \mathrm{l}$. Anion gap was found 
to be $32 \mathrm{mEq} / 1$ (Sodium-[chloride + bicarbonate]). As facilities for blood methanol evaluation were not available, methanol level could not be assessed at that time.

With the following clinical findings, history and evidence of an increased anion gap metabolic acidosis, a provisional diagnosis of acute methanol intoxication was made.

\section{MANAGEMENT}

Patient was transferred to intensive care unit and was treated with humidified oxygen and intravenous fluids. Initially, he was treated with a loading dose of $1.8 \mathrm{ml} / \mathrm{kg} \mathrm{43 \%}$ ethanol (42.7\%) through Ryle's tube, followed by a maintenance dose of $0.2 \mathrm{ml} / \mathrm{kg} / \mathrm{h}$ of $43 \%$ ethanol according to methanol poisoning treatment protocol. Infusion of sodium bicarbonate was started to combat acidosis. Folic Acid tablets were also administered. Reviewing the condition $6 \mathrm{~h}$ later, showed no significant improvement in either clinical or metabolic parameters. Therefore, patient was subjected to urgent hemodialysis twice in the next $24 \mathrm{~h}$ as per treating consultant's advice. $48 \mathrm{~h}$ after admission, patient's clinical condition improved and metabolic acidosis had resolved. He was transferred to the general ward for further treatment. However, his vision had not improved and he was put on high dose steroids (Injection methylprednisolone $1 \mathrm{~g}$ /day) for a week to prevent toxin induced optic neuritis as per advice of consultant ophthalmologist. On day 5, on resuming his daily activity the patient complained of difficulty in opening his shirt buttons and having his food by himself because of trembling of his hands. He also complained having diminished sensation in both legs. Examination revealed absent deep tendon reflexes (both ankle and knee) bilaterally in the lower limb. There was impairment of sensation up to knee bilaterally. The plantar responses were equivocal. Motor system examination showed no gross weakness. Romberg's test was positive. He was also found to have bradykinesia and rigidity during movement. Along with it, he had developed resting tremor in both hands. Speech was slow while memory and intellect were normal. A non-contrast computed tomography (CT) scan of the brain was done and it

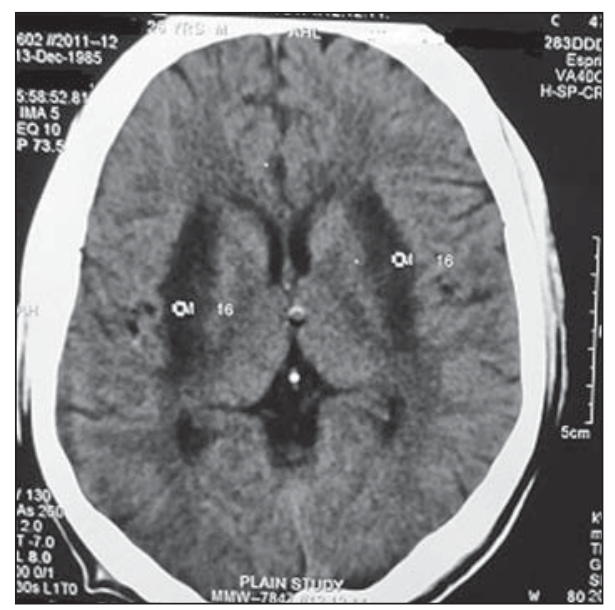

Figure 1: Computed tomography scan showing bilateral putaminal necrosis revealed bilateral symmetrical putaminal necrosis without evidence of hemorrhage [Figures 1 and 2]. A diagnosis of methanol induced parkinsonism and peripheral neuropathy with sensory ataxia was put forth. Cerebrospinal fluid study revealed no abnormality. Nerve conduction study showed evidence of sensory neuropathy. Thyroid profile and serum Vitamin $B_{12}$ level were normal. He was treated with thiamine and other multi vitamin injections. The patient was managed conservatively and was discharged on $15^{\text {th }}$ day of hospitalization with levodopa and multivitamins. Though, patient life was saved due to prompt management, but he suffered from parkinsonian such as disease, peripheral neuropathy and optic neuritis. On 1 month post-discharge follow-up his motor and sensory symptoms had not improved. His vision had slightly improved, but still significant visual impairment persisted.

\section{DISCUSSION}

Methanol poisoning is mostly due to ingestion of methanol containing solvents or liquor and it is a rare, but potentially life-threatening poison. As a solvent, it is present in cleaning solutions, printing and duplicating solutions, adhesives, enamels, stains, dyes, varnishes, thinners and paint removers. ${ }^{[5]}$ It is also widely available as an antifreeze agent. It is also used in preparation of cheap illicit liquor. Methanol is primarily metabolized by the liver. Methanol is initially metabolized to formaldehyde with the aid of enzyme alcohol dehydrogenase and finally to formic acid by the help of aldehyde dehydrogenase. Formic acid is subsequently metabolized to carbon dioxide and water and the step is dependent on the presence of tetrahydrofolate. ${ }^{[5]}$ The knowledge of the metabolic steps has got clinical implications in the treatment of patients, which will be discussed. The toxicities exerted are not primarily due to methanol itself, but due to its toxic metabolites of formaldehyde and formic acid. Formaldehyde has a very short half-life of around 1-2 min and hence there is no accumulation of formaldehyde in plasma. ${ }^{[5]}$ It is therefore formic acid that is responsible for all major clinical symptoms in cases of methanol poisoning. Formic acid inhibits mitochondrial cytochrome oxidase resulting in histotoxic hypoxia.

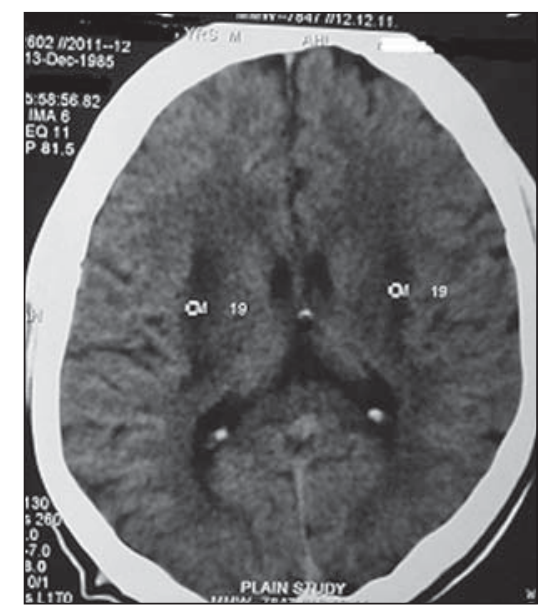

Figure 2: Computed tomography scan showing bilateral putaminal necrosis 
Methanol causes toxicity pertaining to central nervous system (CNS), gastrointestinal and ocular systems. Symptoms of methanol intoxication include nausea, vomiting, abdominal pain, dyspnea, headache, dizziness and visual symptoms including blurring, dimming or blindness, flashes of light, "snowfield" vision, unsteadiness, confusion, coma, seizures and death. Methanol is particularly toxic to the optic nerve and CNS. The pathogenesis is presumed to be histotoxic anoxia in a vascular watershed area, which is the result of direct inhibition of cytochrome oxidase by formic acid. ${ }^{[6]}$ In addition, the increasing pressure following edema in the visual pathway might further aggravate the deterioration due to ischemic changes. ${ }^{[7]}$ The mechanism of subsequent optic atrophy in patients with methanol poisoning is still unknown, it was suggested to be due to progressive demyelination. ${ }^{[8]}$ Optic nerve lesions are considered to be due to myelinoclastic effect of formic acid and due to axonal loss. ${ }^{[9,10]}$

Patients who survive for more than $24 \mathrm{~h}$ show characteristic CT findings of bilateral low attenuation lesions in the putamen and cerebral deep white matter. ${ }^{[9,11]}$ The occipital and frontal lobes are more commonly involved. ${ }^{[12]}$ Putaminal hemorrhage is not uncommon and very rarely this may extend into the ventricular system. Dialysis may increase the risk of hemorrhage. ${ }^{[10]}$ Cerebral cortex involvement is rare. Cerebellar involvement is occasionally seen. It may involve the cortex, white matter and cerebellar nuclei. ${ }^{[13]}$ There is a single reported case of subarachnoid hemorrhage. ${ }^{[14]}$ Whereas the mechanism of cytotoxicity of methanol is well-understood the predilection for damage to deep white matter, retrolaminar optic nerve and putamen is uncertain. In part this distribution of injury appears to relate to the blood supply with these being watershed areas. Poor venous drainage of the putamen has also been proposed. ${ }^{[15]}$ Four signs of methanol intoxication include depressed level of consciousness, altered mental status, decreased visual acuity, visual field defects/scotomata, mydriasis, decreased papillary response, hyperemia of optic discs, retinal edema or glaucoma like cupped/ atrophic discs. Long-term sequel includes optic neuropathy/ blindness, parkinsonism, dystonia, toxic encephalopathy, dementia and axonal poly neuropathy. ${ }^{[16]}$ One hypothesis attributes the predilection for putaminal damage to the pattern of poor venous drainage. Recent hypothesis suggests that the greater susceptibility of optic nerve, macular area of retina and basal ganglia to anoxia and ischemia following methanol intoxication is possibly due to their high metabolic rates. ${ }^{[17]}$ The pathogenesis of the white matter hemorrhagic necrosis remains unexplained. ${ }^{[18]}$ The characteristic clinicopathological findings of methanol intoxication are optic neuropathy, bilateral putaminal hemorrhagic and non-hemorrhagic necrosis. ${ }^{[4]}$ In two other reported cases, CT scans on the $1^{\text {st }}$ day appeared normal, but latter showed putaminal necrosis. ${ }^{[19]}$ Bilateral putaminal necrosis is by no means specific to methanol toxicity and can also be seen in Wilson's disease, Leigh disease, KearnsSayre syndrome, striatal degeneration associated with Leber's optic atrophy, hypoxic-anoxic injuries and carbon monoxide poisoning. ${ }^{[20]}$ However, in carbon monoxide poisoning specific focus of toxicity is the globus pallidus while hypoxic-anoxic injuries additionally involve the caudate nucleus and other central gray nuclei. ${ }^{[20]}$ In methanol intoxication, putaminal necrosis is usually permanent, however in some series significant regression of neurological findings and disappearance of extrapyramidal symptoms are reported. ${ }^{[4]}$ In a follow-up study after a large scale methanol poisoning outbreak, the most common visual disturbances present were optic nerve atrophy, temporal pallor of the optic nerve head, visual field defects and loss of visual acuity (including blindness). The neurological impairment were polyneuropathy, encephalopathy, ataxia and peripheral sensory neuropathy involving legs. ${ }^{[1]}$ Cases of acute onset parkinsonism following methanol poisoning have been described. ${ }^{[2]}$

The treatment is usually aimed at prompt elimination of methanol and prevention of complications. Ethanol and fomepizole competitively inhibits alcohol dehydrogenase and retards ethanol metabolism and accumulation of its metabolites. Folic acid and folinic acid enhances metabolism of toxic formic acid to non-toxic carbon dioxide and water. Supportive measures include management of acidosis, coma, seizure and encephalopathy and airway maintenance. Hemodialysis is used ultimately when other therapeutic measures fail. Complications such as optic neuritis, polyneuropathy and parkinsonism needs special mention. Funduscopy and CT scan must be done to look for these potential complications. High dose steroids are generally used for treating optic neuritis ${ }^{[7]}$ while levodopa has been tried with variable success in cases of methanol induced parkinsonism. ${ }^{[2]}$

\section{CONCLUSION}

This case elucidates severe neurological toxicities associated with cases of methanol poisoning. The particular patient unfortunately developed optic neuritis, acute parkinsonism such as syndrome, peripheral neuropathy and ataxia which all are rare, but reported toxicities of methanol poisoning. Clinicians should note that the diagnosis of methanol poisoning should be borne in mind when evaluating patients with unexplained positive anion gap metabolic acidosis even if the patient's past medical history is not suggestive. Such a diagnosis may then be confirmed with evidence of CT scan showing putaminal necrosis and optic neuritis. Hence, treatment should commence at earliest to minimize complications.

\section{REFERENCES}

1. Paasma R, Hovda KE, Jacobsen D. Methanol poisoning and long term sequelae-A six years follow-up after a large methanol outbreak. BMC Clin Pharmacol 2009;9:5.

2. Reddy NJ, Lewis LD, Gardner TB, Osterling W, Eskey CJ, Nierenberg DW. Two cases of rapid onset Parkinson's syndrome following toxic ingestion of ethylene glycol and methanol. Clin Pharmacol Ther 2007;81:114-21.

3. Jarwani BS, Motiani P, Divetia R, Thakkar G. Rare combination of bilateral putaminal necrosis, optic neuritis, and polyneuropathy in a case of acute methanol intoxication among patients met with hooch tragedy in Gujarat, India. J Emerg Trauma Shock 2012;5:356-9.

4. Hantson P, Duprez T, Mahieu P. Neurotoxicity to the basal ganglia shown by magnetic resonance imaging (MRI) following poisoning by methanol and other substances. J Toxicol Clin Toxicol 1997;35:151-61.

5. Barceloux DG, Bond GR, Krenzelok EP, Cooper H, Vale JA, American Academy of Clinical Toxicology Ad Hoc Committee on the Treatment 
Guidelines for Methanol Poisoning. American Academy of Clinical Toxicology practice guidelines on the treatment of methanol poisoning. J Toxicol Clin Toxicol 2002;40:415-46.

6. Hayreh MS, Hayreh SS, Baumbach GL, Cancilla P, Martin-Amat G, Tephly TR, et al. Methyl alcohol poisoning III. Ocular toxicity. Arch Ophthalmol 1977;95:1851-8.

7. Sodhi PK, Goyal JL, Mehta DK. Methanol-induced optic neuropathy: Treatment with intravenous high dose steroids. Int $\mathrm{J}$ Clin Pract 2001;55:599-602.

8. Sharma M, Volpe NJ, Dreyer EB. Methanol-induced optic nerve cupping. Arch Ophthalmol 1999;117:286

9. Hsu HH, Chen CY, Chen FH, Lee CC, Chou TY, Zimmerman RA. Optic atrophy and cerebral infarcts caused by methanol intoxication: MRI. Neuroradiology 1997;39:192-4.

10. Phang PT, Passerini L, Mielke B, Berendt R, King EG. Brain hemorrhage associated with methanol poisoning. Crit Care Med 1988;16:137-40

11. Patankar T, Bichile L, Karnad D, Prasad S, Rathod K. Methanol poisoning: Brain computed tomography scan findings in four patients. Australas Radiol 1999;43:526-8.

12. Gaul HP, Wallace CJ, Auer RN, Fong TC. MR findings in methanol intoxication. AJNR Am J Neuroradiol 1995;16:1783-6.

13. Rubinstein D, Escott E, Kelly JP. Methanol intoxication with putaminal and white matter necrosis: MR and CT findings. AJNR Am J Neuroradiol 1995;16:1492-4.
14. del Carpio-O'Donovan R, Glay J. Subarachnoid hemorrhage resulting from methanol intoxication: Demonstration by computed tomography. Can Assoc Radiol J 1992;43:299-301.

15. McLean DR, Jacobs $H$, Mielke BW. Methanol poisoning: A clinical and pathological study. Ann Neurol 1980;8:161-7.

16. Kocakoc E, Kiris A, Ozdemir H, Arslan A, Bozgeyik Z. Putaminal necrosis due to methanol intoxication: early MRI findings, \{Online\} http://www. eurorad.org/case. .php?id=1670

17. Sharpe JA, Hostovski M, Bilbao JM, Rewcastle NB. Methanol optic neuropathy: a histopatologic study. Neurology 1982;32:393-408.

18. Mc Lean DR, Jacobs $H$, Mielke BW. Basal ganglia lesions in methanol poisoning. MR appearance. J Comput Assist Tomogr 1988;12:168-9.

19. LeWitt PA, Martin SD. Dystonia and hypokinesis with putaminal necrosis after methanol intoxication. Clin Neuropharmacol 1988;11:161-7.

20. Koopmans RA, Li DK, Paty DW. Basal ganglia lesions in methanol poisoning: MR appearance. J Comput Assist Tomogr 1988;12:168-9.

How to cite this article: Mangaraj S, Sethy G, Sen RK, Rout RN. Methanol poisoning induced acute onset Parkinsonism, optic neuritis and peripheral neuropathy in a patient. Int $\mathrm{J}$ Med Public Health 2014;4:130-3.

Source of Support: Nil. Conflict of Interest: None declared. 